\title{
All-optical 3D atomic loops generated with Bessel light fields
}

\author{
Karen Volke-Sepúlveda ${ }^{1,}$ * and Rocío Jáuregui ${ }^{1}$, \\ ${ }^{1}$ Departamento de Física Teórica, Instituto de Física, \\ Universidad Nacional Autónoma de México, A.P. 20-364, México 01000 D.F. México
}

(Dated: September 15, 2021)

\begin{abstract}
The propagation invariance of Bessel beams as well as their transversal structure are used to perform a comparative analysis of their effect on cold atoms for four different configurations and combinations thereof. We show that, even at temperatures for which the classical description of the atom center of mass motion is valid, the interchange of momentum, energy and orbital angular momentum between light and atoms yields efficient tools for all-optical trapping, transporting and, in general, manipulating the state of motion of cold atoms.

PACS numbers: 37.10.Gh, 37.10.Jk, 42.50.Tx
\end{abstract}

\section{INTRODUCTION}

Over the last decade, experiments on the interaction between light and cold atoms have seen tremendous advances. Laser cooling of neutral atoms is nowadays a well-established procedure and solid steps for novel experiments in research areas such as atom optics and quantum information processing with atomic systems have been taken. During the last fifteen years, the development of far off resonance traps (FORT's) [1] has allowed the organization of cold matter in optical lattices [2] and, with this, the study of single particle Bloch physics. The creation of trapped degenerate atomic gases, on the other hand, is one of the most exciting scientific achievements of modern times [3], as it has opened, for instance, the possibility of realizing interference of matter waves [4].

In these areas, the use of light beams with especial intensity and/or phase structure yielding peculiar dynamical properties, plays a very important role. Bessel beams [5], for instance, have been proposed as waveguides for atom transport due to their propagation invariance [6, 7]. The measurement of the mechanical properties of Mathieu beams could be performed through the analysis of their effects on cold atoms [8. The transfer of orbital angular momentum (OAM) from Laguerre-Gaussian (LG) laser modes [9, 10, or high-order Bessel beams [11, 12, 13] to cold matter has been the subject of theoretical studies in both, paraxial and non-paraxial regimes. For the case of a LG beam interacting with a diatomic molecule, it was found that OAM has in general a weak effect on the internal state, since it becomes relevant at the electric quadrupole interaction level, while the major mechanism of exchange occurs in the electric dipole approximation and involves only the center of mass motion [10. For non-paraxial Bessel beams interacting with a single atom, in contrast, the probability that the internal state of an atom acquires orbital angular momentum from light is maximum when the atom is located at the beam axis [13. In fact the helicity factor $k_{z} c / \omega$, which is related to the projection of angular momentum along the main direction of propagation, could be used to directly enhance or suppress atomic transitions [14].

The first experimental demonstration of OAM transfer to cold atoms was reported almost a decade ago by Tabosa and coworkers [15] and, very recently, OAM was transferred to a Bose Einstein condensate [16]. OAM transfer is also an important aspect in the study of circular optical lattices and helical waveguides, which are interesting alternatives for interference experiments with matter waves and quantum transport. For example, Haroutyunyan and Nienhuis [17. have recently explored the use of stationary waves in the angular direction, generated by the superposition of two counter rotating LG beams propagating in the same direction, as a more efficient alternative for achieving the exchange of angular momentum between light and cold atoms. In this case, the confinement in the radial direction is achieved through an extra trapping potential with cylindrical symmetry, but the dynamics along the $z$ axis is completely free. A circular optical potential of this kind would split the wave function of a single localized atom into clockwise and anticlockwise components, which may interfere under certain confinement conditions [17. Bhattacharya [18] on the other hand, presented a simplified analysis of a curved helical lattice as an atom guide, which could be generated by the superposition of two identical LG beams propagating in opposite directions. This motivated, in turn, a study on the possibility of having bound states in such curved helical potentials [19. Circular and rotating optical lattices have been studied as well, in the context of condensed matter and many particle systems, such as Fermi gases and Bose

*Electronic address: karen@fisica.unam.mx

${ }^{\dagger}$ Electronic address: rocio@fisica.unam.mx 
Einstein condensates [20]-24]. Moreover, ring shaped optical lattices represent appropriated potentials for studying quasi one dimensional physical systems with closed boundary conditions [21, 22]. Most of these systems consider, besides optical fields, external magnetic fields to achieve confinement in one or more spatial dimensions.

In this work we perform a theoretical comparative analysis of the effect of four different light fields of circular structure on a dilute gas of cold atoms whenever the effect of collisions among them can be neglected. In all cases, we use Bessel beams (BB) and superpositions thereof, taking advantage of their propagation invariance property. First, we analyze the case of a single high order BB. Secondly, we study the case of an optical field with $2 m$ intertwined helicoidal lines of light, similar to the curved helical waveguides studied by Bhattacharya [18], but in this case, it results from superimposing two identical BBs propagating in opposite directions. In the third place, we look at a three dimensional circular lattice, corresponding to the simultaneous generation of standing waves in the radial, angular and axial directions. As a fourth option, we analyze a circular optical lattice constituted by a collection of individual toroidal traps along the $z$-axis, which can be achieved by interfering two counter propagating BBs of opposite helicity. Our interest in these particular configurations arises from the fact that they can be combined and used successively for creating 'atom loops' in predesigned ways, as it will be demonstrated in the last section of the paper. Our approach follows the semiclassical description made by Gordon and Ashkin [25, which can be applied when the atom velocity is sufficiently low but not beyond the quantum limits. The quantum mechanics treatment will be presented elsewhere.

\section{ROTATING LIGHT BEAMS AND CIRCULAR LATTICES}

As a starting point for the present discussion, we will briefly describe the main properties of a BB within a vectorial treatment, in order to account for polarization properties. Under ideal conditions, the electromagnetic field of a Bessel mode has cylindrical symmetry which guarantees its propagation invariance along the $z$-axis:

$$
\begin{aligned}
E_{\rho}^{\kappa} & =\frac{\partial^{2}}{\partial z \partial \rho} \Pi_{\kappa}^{(T M)}-\frac{1}{\rho} \frac{\partial^{2}}{c \partial t \partial \phi} \Pi_{\kappa}^{(T E)} \\
E_{\phi}^{\kappa} & =\frac{1}{\rho} \frac{\partial^{2}}{\partial z \partial \phi} \Pi_{\kappa}^{(T M)}+\frac{\partial^{2}}{c \partial t \partial \rho} \Pi_{\kappa}^{(T E)}, \\
E_{z}^{\kappa} & =-\frac{\partial^{2}}{c^{2} \partial t^{2}} \Pi_{\kappa}^{(T M)}+\frac{\partial^{2}}{\partial z^{2}} \Pi_{\kappa}^{(T M)}
\end{aligned}
$$

where $\Pi_{\kappa}^{j}=\mathcal{E}_{\kappa}^{j} \psi_{m}\left(k_{\perp} \rho, \phi\right) e^{i\left(k_{z} z-\omega t\right)}$, with $\psi_{m}\left(k_{\perp} \rho, \phi\right)=J_{m}\left(k_{\perp} \rho\right) e^{i m \phi} . J_{m}$ is the Bessel function of order $m$ and $\mathcal{E}^{(T E)}\left(\mathcal{E}^{(T M)}\right)$ is proportional to the amplitude of the transverse electric (magnetic) mode. $\kappa$ denotes collectively the parameters that define the mode, that is, the propagation wavenumber along the $z$ axis, $k_{z}$, the transverse propagation wavenumber $k_{\perp}$ and the azimuthal index $m$.

By superpositions of TE and TM Bessel modes, different polarizations states can be obtained. In the literature [11, 26, 27] the modes

$$
\begin{aligned}
& \vec{E}_{m}^{(\mathcal{L})}\left(\vec{r}, t ; k_{\perp}, k_{z}\right)=E_{0}^{(\mathcal{L})}\left[\left(\hat{e}_{x}+i \hat{e}_{y}\right) \psi_{m}-\frac{i}{2}\left(\frac{k_{\perp}}{k_{z}}\right) \psi_{m+1} \hat{e}_{z}\right] \\
& \vec{E}_{m}^{(\mathcal{R})}\left(\vec{r}, t ; k_{\perp}, k_{z}\right)=E_{0}^{(\mathcal{R})}\left[\left(\hat{e}_{x}-i \hat{e}_{y}\right) \psi_{m}+\frac{i}{2}\left(\frac{k_{\perp}}{k_{z}}\right) \psi_{m-1} \hat{e}_{z}\right]
\end{aligned}
$$

are considered to be the analogues of left $(\mathcal{L})$ and right-handed $(\mathcal{R})$ circularly polarized plane wave modes. Their superpositions $\mathbf{E}_{m}^{(\mathcal{R})} \pm \mathbf{E}_{m}^{(\mathcal{L})}$ define linearly polarized modes. In terms of TE and TM modes,

$$
\begin{aligned}
\vec{E}_{m}^{(\mathcal{L})} & =E_{0}^{(\mathcal{L}) \prime}\left(\vec{E}_{m+1}^{(T M)}-i \frac{c k_{z}}{\omega} \vec{E}_{m+1}^{(T E)}\right) \\
\vec{E}_{m}^{(\mathcal{R})} & =E_{0}^{(\mathcal{R}) \prime}\left(\vec{E}_{m-1}^{(T M)}+i \frac{c k_{z}}{\omega} \vec{E}_{m-1}^{(T E)}\right) .
\end{aligned}
$$

The mechanical properties of the photons associated with Bessel modes are directly related to the numbers $\omega, k_{z}, m$ that characterize them, along with the polarization. In fact, $\hbar \omega, \hbar k_{z}, m \hbar$ correspond to the energy, linear momentum and orbital angular momentum along the $z$ direction respectively [28]. A linearly polarized mode has the structure

$$
\vec{E}_{m}\left(\vec{r}, t ; k_{\perp}, k_{z}\right)=E_{0}\left[\left(\psi_{m} \hat{e}_{x}-\frac{i}{2}\left(\frac{k_{\perp}}{k_{z}}\right)\left(\psi_{m+1}-\psi_{m-1}\right) \hat{e}_{z}\right]\right.
$$




\section{(a) Transverse cross section}
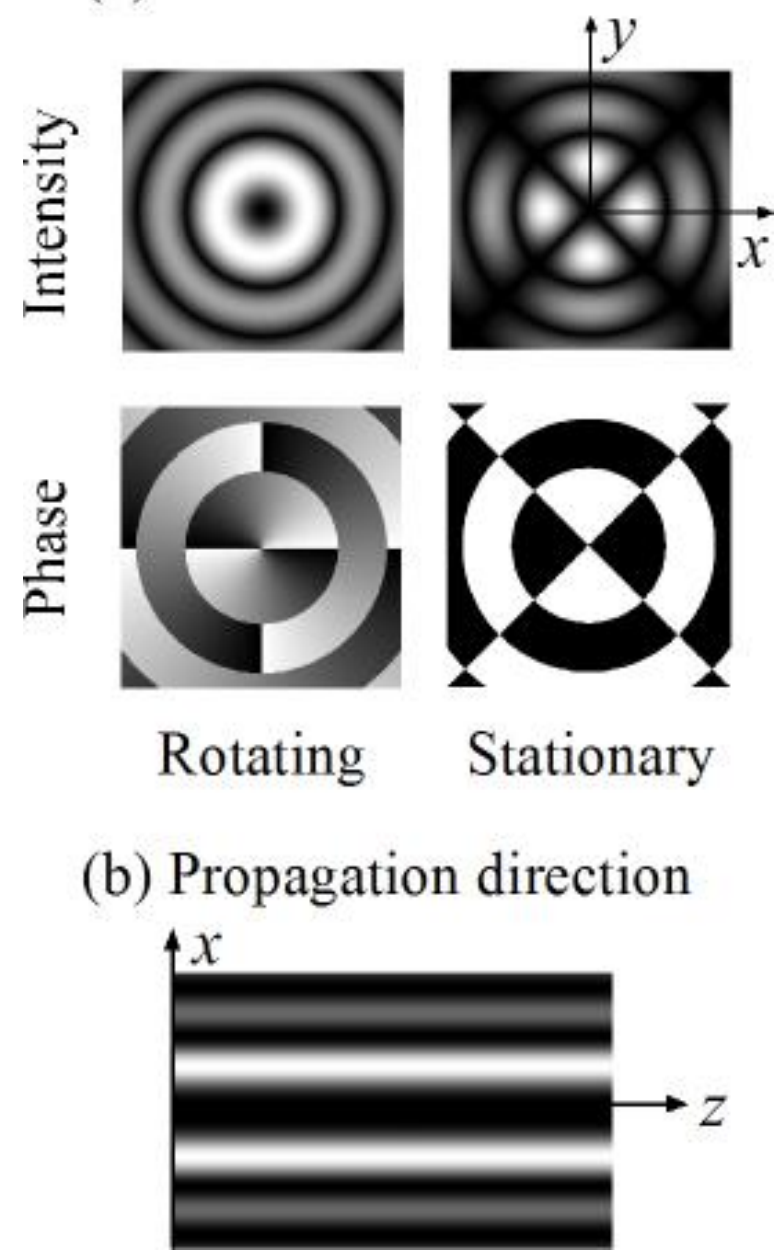

FIG. 1: Comparison between (a) a rotating and (b) a stationary Bessel beam of order $m=2$. The phase values for both beams are indicated in the color bar on the right hand side, in units of $\pi$ radians. Along the propagation axis, both beams have a similar behavior due to their ideal propagation invariance.

In what follows, we will describe in some detail each one of the four optical fields of interest, providing an explicit analytical expression and a brief discussion about their experimental generation. For this purpose, it will be useful to establish first a distinction between rotating and stationary BBs.

While a linearly polarized rotating BB corresponds to that given by Eq. (6), with $\psi_{m}(\rho, \varphi)=J_{m}\left(k_{\perp} \rho\right) e^{i m \varphi}$, a stationary BB is formed by the superposition of two rotating BBs of the same topological charge $|m|$ traveling along the same axis and direction, but rotating in opposite sense $( \pm m)$, giving rise to

$$
\begin{aligned}
& \vec{E}_{m}(\vec{r}, t)=E_{0} e^{i\left(k_{z} z-\omega t-\varphi_{0}\right)}\left[J_{m}\left(k_{\perp} \rho\right) \cos \left(m \varphi+\varphi_{0}\right) \hat{e}_{x}\right. \\
& \left.-\frac{i}{2} \frac{k_{\perp}}{k_{z}}\left(J_{m+1}\left(k_{\perp} \rho\right) \cos \left[(m+1) \varphi+\varphi_{0}\right]-J_{m-1} \cos \left[(m-1)\left(k_{\perp} \rho\right) \varphi+\varphi_{0}\right]\right) \hat{e}_{z}\right] .
\end{aligned}
$$

Here and in the following, $\varphi_{0}=0\left(\varphi_{0}=\pi / 2\right)$ stands for even (odd) values of $m$. Figure 1(a) illustrates the intensity and phase distributions of an ideal rotating BB, while Figure 1(b) shows the same for an ideal stationary BB, in both cases $m=2$. It is seen that the ideal fields exhibit propagation invariance of their intensity along the $z$ axis.

The optical fields of interest will be constructed as superpositions of linearly polarized Bessel modes in the sense discussed above, either rotating or stationary, with the transverse component of their electric fields oriented along the $x$ axis.

Case 1. Single rotating Bessel beam. 


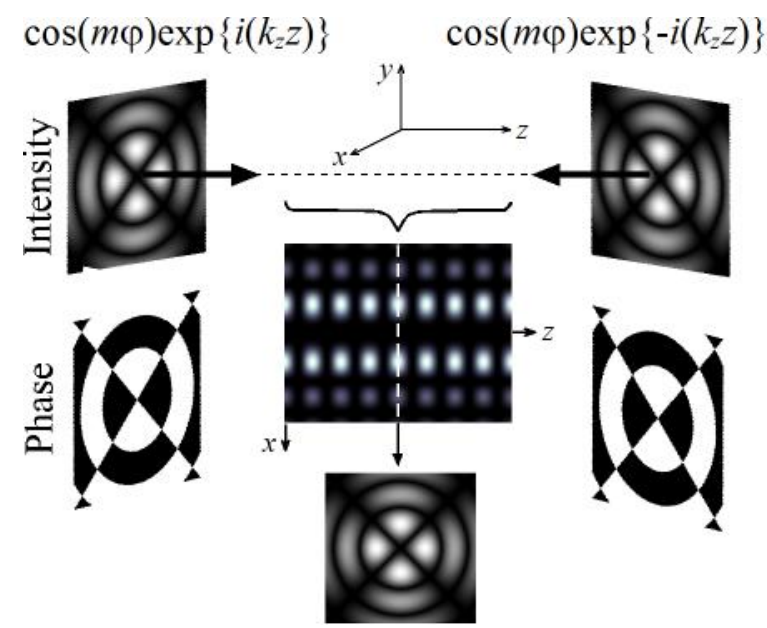

FIG. 2: (Color online) Schematic of the superposition of two rotating BBs propagating in opposite directions; the rotation sense of the beams is opposite as well with respect to the same fixed reference frame. Transverse cross sections of the resulting field at different $z$ planes indicate an intensity distribution that is twisted around the $z$ axis, but stationary in time. The whole structure resembles a rope with $2 m$ main inner strands of light twisted together, and outer groups of strands with reduced intensity.

A single BB in interaction with cold atoms has been studied before [6, 7, 13. Here we include this simple case for comparative purposes with the other configurations and also to emphasize some of its applications for controlling atomic motion. The expression for a linearly polarized rotating BB is, according to Eq. (6),

$$
\vec{E}_{m}^{(1)}\left(\vec{r}, t ; k_{\perp}, k_{z}\right)=E_{0} e^{i\left(k_{z} z+m \varphi-\omega t\right)}\left[J_{m}\left(k_{\perp} \rho\right) \hat{e}_{x}-\frac{i}{2} \frac{k_{\perp}}{k_{z}}\left(J_{m+1}\left(k_{\perp} \rho\right) e^{i \varphi}-J_{m-1}\left(k_{\perp} \rho\right) e^{-i \varphi}\right) \hat{e}_{z}\right] .
$$

Experimentally, reasonable approximations to BBs of different orders have been efficiently generated by illuminating an axicon or conical lens with a single-ringed Laguerre-Gaussian mode of order $m$ [29. Another approach is to obtain the desired BB directly from properly designed computer generated holograms (CGH) [30, which can be displayed in spatial light modulators (SLM) [31. The original setup proposed by Durnin and coworkers [5, consisting of a dark screen with a thin annulus transmitance function placed at the back focal plane of a positive lens, turns out to be inefficient for optical trapping experiments, although it is the best approximation to the theoretical expression. In all cases, of course, BBs can be generated only within a finite region and, under current experimental conditions, the paraxial approximation is generally fulfilled. It is worth to mention, however, that BBs with relatively large transverse dimensions $\left(k_{\perp} / k_{z}<<1\right)$ can be reduced with additional lenses in order to make them more suitable for atom trapping experiments.

Case 2. Twisted helical lattice.

This field can be generated by the interference of two rotating BBs with the same helicity but propagating in opposite directions. This means that the two beams have the same projection of their respective angular momenta along their own propagation direction but, with respect to the same and fixed reference frame, they are rotating in opposite directions, as illustrated in Fig. 2. The resulting field is described by

$$
\begin{aligned}
\vec{E}_{m}^{(2)}(\vec{r}, t) & =E_{0} e^{-i\left(\omega t+\varphi_{0}\right)}\left[J_{m}\left(k_{\perp} \rho\right) \cos \left(m \varphi+k_{z} z+\varphi_{0}\right) \hat{e}_{x}\right. \\
& \left.-\frac{i}{2}\left(\frac{k_{\perp}}{k_{z}}\right)\left(J_{m+1}\left(k_{\perp} \rho\right) \cos \left[(m+1) \varphi+k_{z} z+\varphi_{0}\right]-J_{m-1}\left(k_{\perp} \rho\right) \cos \left[(m-1) \varphi+k_{z} z+\varphi_{0}\right]\right) \hat{e}_{z}\right]
\end{aligned}
$$

The experimental generation of this optical field can be performed by introducing a rotating BB into an amplitude division interferometer; each portion of the split beam should suffer the same number of reflections, so that the helicity is preserved for both of them before being superimposed again along the same axis while propagating in opposite directions. 


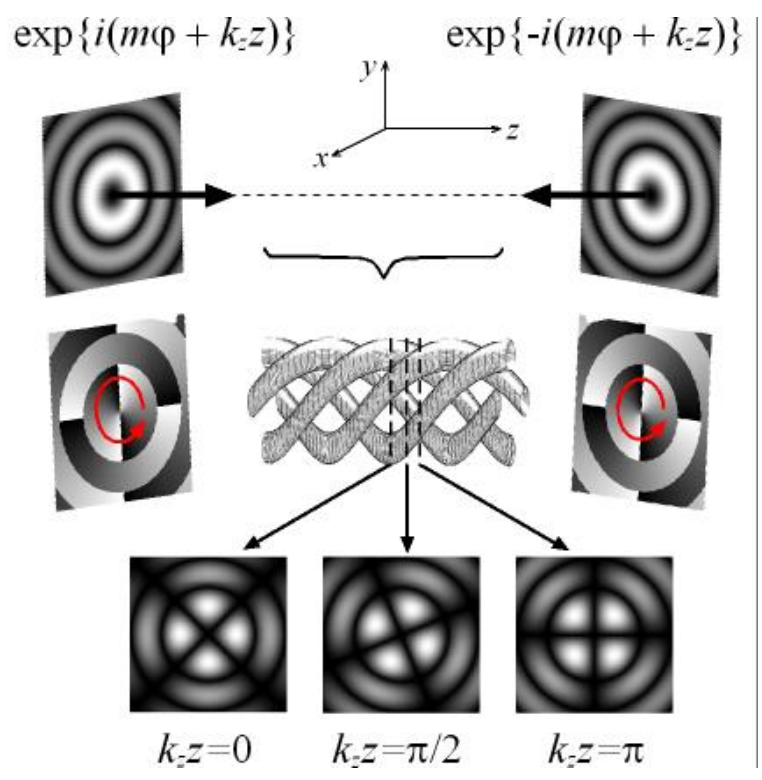

FIG. 3: Schematic of the superposition of two stationary BBs propagating in opposite directions. Standing waves are generated in the three spatial directions: radial, angular and axial.

Case 3. 3D stationary circular lattice.

In this case, we consider the interference of two stationary Bessel modes of the type described by Eq. (7), but propagating in opposite directions along the same $z$ axis. The resulting optical field will exhibit standing waves in all the three spatial dimensions within a circular cylindrical geometry,

$$
\begin{aligned}
\vec{E}_{m}^{(3)}(\vec{r}, t) & =E_{0} e^{-i\left(\omega t+\varphi_{0}\right)} \cos \left(k_{z} z\right)\left[J_{m}\left(k_{\perp} \rho\right) \cos \left(m \varphi+\varphi_{0}\right) \hat{e}_{x}\right. \\
& \left.-\frac{i}{2} \frac{k_{\perp}}{k_{z}}\left(J_{m+1}\left(k_{\perp} \rho\right) \cos \left[(m+1) \varphi+\varphi_{0}\right]-J_{m-1}\left(k_{\perp} \rho\right) \cos \left[(m-1) \varphi+\varphi_{0}\right]\right) \hat{e}_{z}\right]
\end{aligned}
$$

Intensity nodal surfaces correspond, along the radial direction, to concentric dark cylinders whose radii $\rho=\rho_{m n}$, are defined by $k_{\perp} \rho_{m n}=x_{n m}$, with $x_{m n}$ the $n$-th root of the Bessel function of order $m$. The cylinders are intersected by $2|m|$ semi-infinite nodal planes along the azimuthal coordinate, defined by $\left[|m| \varphi_{n}+\varphi_{0}\right]=(2 n-1) \pi / 2$, where $n=1,2, \ldots, 2|m|$. Finally, there are also nodal planes along the $z$ axis corresponding to $z_{n}=(2 n-1) \lambda_{z} / 4$, with $n$ an integer and $\lambda_{z}=2 \pi / k_{z}$. The experimental generation of a lattice like this may involve two steps. First, it is necessary to obtain the stationary $\mathrm{BB}$, which can be done either directly, by means of CGH, or by interfering two counter rotating BBs propagating along the same axis and direction, for instance. Once obtained the stationary BB, an amplitude division interferometer would be appropriated for superimposing two equally weighted portions of it, aligned along the same axis, but propagating in opposite directions. This is schematically illustrated in Fig. 3.

Case 4. Toroidal train lattice.

A set of toroidal traps along an axis can be generated by the interference of two rotating BBs with opposite helicities and propagating in opposite directions. This means that the two beams have opposite projections of their respective angular momenta along their own propagation direction but, with respect to the same reference frame, they are rotating in the same direction, as illustrated in Fig. 4. The resulting field is given by

$$
\begin{aligned}
\vec{E}_{m}^{(4)}(\vec{r}, t) & =E_{0} e^{i(m \varphi-\omega t)} \cos \left(k_{z} z\right)\left[\hat{e}_{x} J_{m}\left(k_{\perp} \rho\right)\right. \\
& \left.-\frac{i}{2}\left(\frac{k_{\perp}}{k_{z}}\right)\left[J_{m+1}\left(k_{\perp} \rho\right) e^{i \varphi}-J_{m-1}\left(k_{\perp} \rho\right) e^{-i \varphi}\right] \hat{e}_{z}\right]
\end{aligned}
$$

A transverse cross section of this field at an antinodal plane along the $z$ axis is exactly the same that the transverse cross section of a propagating BB, but null intensity occurs at the $z$ nodal planes which, as in Case 3, correspond 


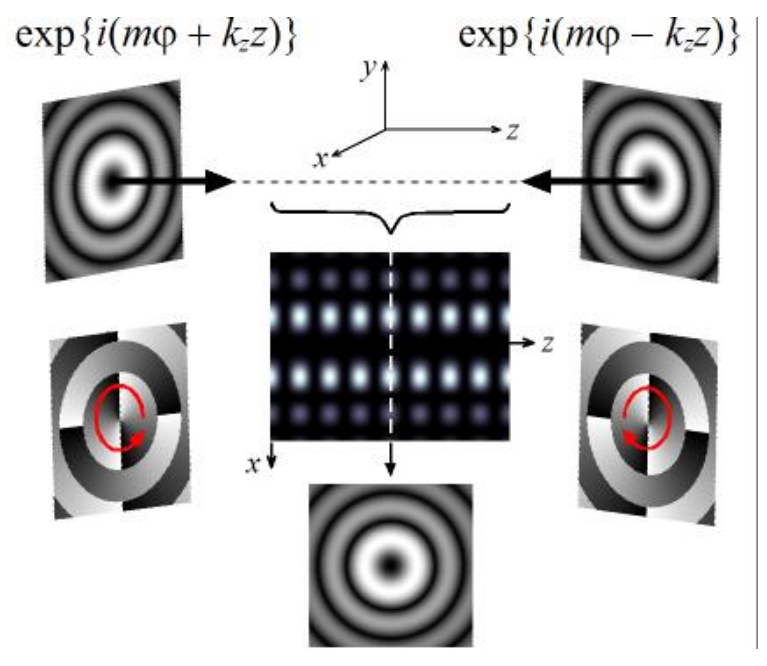

FIG. 4: (Color online) Schematic of the superposition of two rotating BBs propagating in opposite directions; the rotation sense of the beams in this case is the same with respect to the same fixed reference frame. The 3D intensity distribution would resemble a straight backbone of light.

to the planes $z=(2 n-1) \lambda_{z} / 4$ ( $n$ integer). The experimental generation of this optical field can be performed by introducing a rotating BB into an amplitude division interferometer; one portion of the split beam should suffer an extra reflection, so that its helicity is inverted with respect to the other portion of the beam before joining them together along the same axis while propagating in opposite directions.

As we shall see in the next section, Bessel optical modes, either stationary or propagating, exhibit interesting features in interaction with cold atoms, due to their propagation invariance property and their multiringed radial structure.

\section{SEMICLASSICAL DESCRIPTION OF A SINGLE ATOM MOTION WITHIN THE LIGHT FIELD}

We take the standard semiclassical description as in the pioneer works by Letokhov and coworkers 32 and Gordon and Ashkin 25. In this approximation, a monochromatic electromagnetic wave describable by a coherent state couples to the dipole moment of an atom. This dipole moment $\vec{\mu}_{12}$ is related to the electromagnetic transitions between the atom levels that, for simplicity, will be taken to have just two accessible options. The coupling $g=i \vec{\mu}_{12} \cdot \vec{E} / \hbar$, depends explicitly on the orientation of the electric field $\vec{E}$ of the wave. For the systems described in this work, $\vec{E}$ arises from linearly polarized beams and has a longitudinal component that is much smaller than the transverse component since $k_{z} \gg k_{\perp}$. As a consequence, from now on, the effect of longitudinal fields on the atom will be neglected, i.e., $g \sim i \vec{\mu}_{12} \cdot \vec{E}_{\perp} / \hbar=\left[i\left(\vec{\mu}_{12} \cdot \mathbf{e}_{x}\right) E_{0} / \hbar\right] \tilde{g}(\rho, \varphi, z)$, where $\tilde{g}$ contains the information about the spatial structure of the light field.

In the following, we shall describe the atom motion under the assumption that its kinetic energy is low enough to be sensitive to the optical force but large enough to admit a classical description in terms of Newton equations. The expression for the average semiclassical velocity-dependent force [25], valid for both propagating and standing beams, is:

$$
\langle\vec{f}\rangle=\hbar \tilde{\Gamma} p^{\prime}\left[\left[(\vec{v} \cdot \vec{\alpha})(1-p)(1+p)^{-1}+\Gamma / 2\right] \vec{\beta}+[(\vec{v} \cdot \vec{\beta})-\delta \omega] \vec{\alpha}\right]
$$

with

$$
\tilde{\Gamma}=\Gamma /\left[\Gamma\left(1+p^{\prime}\right)+2 \vec{v} \cdot \vec{\alpha}\left[1-p / p^{\prime}-p\right]\left[p^{\prime} /(1+p)\right]\right],
$$

$\Gamma=4 k^{3}\left|\vec{\mu}_{12}\right|^{2} / 3 \hbar$ the Einstein coefficient, $\delta \omega=\omega-\omega_{0}$ the detuning between the wave frequency $\omega$ and the transition frequency $\omega_{0}, p=2|g| /{ }^{2}\left((\Gamma / 2)^{2}+\delta \omega^{2}\right)$ a parameter linked to the difference $D$ between the populations of the two levels of the atom, $D=1 /(1+p)$, and finally $p^{\prime}=2|g|^{2} /\left|\gamma^{\prime}\right|^{2}$, with $\gamma^{\prime}=(\vec{v} \cdot \vec{\alpha})(1-p)(1+p)^{-1}=\Gamma / 2+i(-\delta \omega+(\vec{v} \cdot \vec{\beta}))$. The spatial structure of a beam enters basically in the coupling factor $g=|g| e^{i \phi}$, which appears in $p$ and $p^{\prime}$, and defines the vectors $\vec{\alpha}$ and $\vec{\beta}$ through the equation $\nabla g=(\vec{\alpha}+i \vec{\beta}) g$, leading to $\vec{\alpha}=\vec{\nabla} \ln |g|$ and $\vec{\beta}=\vec{\nabla} \phi$. 


\begin{tabular}{|c|c|c|c|}
\hline Case & $\tilde{g}$ & $\vec{\alpha}$ & $\vec{\beta}$ \\
\hline 1 & $J_{m}\left(k_{\perp} \rho\right) e^{i\left(k_{z} z-m \varphi\right)}$ & $k_{\perp} \frac{J_{m}^{\prime}\left(k_{\perp} \rho\right)}{J_{m}\left(k_{\perp} \rho\right)} \hat{e}_{\rho}$ & $\frac{m}{\rho} \hat{e}_{\varphi}+k_{z} \hat{e}_{z}$ \\
\hline 2 & $J_{m}\left(k_{\perp} \rho\right) \cos \left(k_{z} z+m \varphi\right)$ & $k_{\perp} \frac{J_{m}^{\prime}\left(k_{\perp} \rho\right)}{J_{m}\left(k_{\perp} \rho\right)} \hat{e}_{\rho}-\left(\frac{m}{\rho} \hat{e}_{\varphi}+k_{z} \hat{e}_{z}\right) \tan \left(m \varphi+k_{z} z\right)$ & $\overrightarrow{0}$ \\
\hline 3 & $J_{m}\left(k_{\perp} \rho\right) \cos \left(k_{z} z\right) \cos (m \varphi)$ & $k_{\perp} \frac{J_{m}^{\prime}\left(k_{\perp} \rho\right)}{J_{m}\left(k_{\perp} \rho\right)} \hat{e}_{\rho}-\frac{m}{\rho} \tan (m \varphi) \hat{e}_{\varphi}-\tan \left(k_{z} z\right) \hat{e}_{z}$ & $\overrightarrow{0}$ \\
\hline 4 & $J_{m}\left(k_{\perp} \rho\right) \cos \left(k_{z} z\right) e^{i m \varphi}$ & $k_{\perp} \frac{J_{m}^{\prime}\left(k_{\perp} \rho\right)}{J_{m}\left(k_{\perp} \rho\right)} \hat{e}_{\rho}-k_{z} \tan \left(k_{z} z\right) \hat{e}_{z}$ & $\frac{m}{\rho} \hat{e}_{\varphi}$ \\
\hline
\end{tabular}

TABLE I: Coupling factor $\tilde{g}$, and conservative $\vec{\alpha}$ and dissipative $\vec{\beta}$ vectors defining the atom-BB interaction, Eq. $\sqrt{12}$ for the different beam configurations described in section 2 .

In experiments with cold atoms, it is well known that gravity effects should, in general, be taken into account to describe accurately their motion. Here, we will consider that the $z$ axis of the light field configurations is oriented along the vertical direction. The atoms are downloaded to the optical trap, with most of their kinetic energy coming from the axial velocity which is assumed, unless otherwise stated, to be negative.

In Table I, the spatial structure factor $\tilde{g}(\rho, \varphi, z)$ associated with the different beam configurations is given along with the force factors $\vec{\alpha}$ and $\vec{\beta}$.

\section{NUMERICAL RESULTS}

In this work we shall consider red detuned far off resonance light beams. The bright regions of the light intensity distribution correspond to minima of the effective potential energy $V_{\text {eff }}$ associated with the term $\vec{\alpha}=-\vec{\nabla} V_{\text {eff }}=$ $\vec{\nabla} \ln |g|$. The behavior of the atom in the light field depends not only on its initial balance between kinetic and effective potential energy, but also on its initial momentum and position. In all the studied cases, we will illustrate the behavior of an atomic cloud, which means that we will show the paths of several atoms whose initial conditions vary within a certain range of experimentally accessible values.

Unless otherwise stated, the parameters in the numerical simulations consider ${ }^{85} \mathrm{Rb}$ atoms. Following Ref. [1, the laser beam is considered with a detuning of $67 \mathrm{~nm}$ to the red of the $5{ }^{2} S_{1 / 2}-5{ }^{2} P_{1 / 2}$ transition at $795 \mathrm{~nm}$ and an irradiance of $6 \mathrm{~mW} / \mu \mathrm{m}^{2}$ that determines the value of the coupling constant $\left|\vec{\mu}_{12} \cdot E_{0} \hat{e}_{x}\right| / \hbar$. The trajectories of the atoms are described taking the laser wavelength as unit of length and, as unit of time, the inverse of the Einstein coefficient $\Gamma$ which, for $5^{2} P_{1 / 2}$ state of ${ }^{85} \mathrm{Rb}$, is $3.7 \times 10^{7} \mathrm{~s}^{-1}$. The initial kinetic energies are reported in terms of the corresponding "temperature" by dividing by the Boltzmann constant $k_{B}$. Although we have analyzed several values of the light field characteristic parameters, in order to be specific we report just the results where $k_{z}=0.995 \omega / c$ and the topological charge $m=2$. This makes a paraxial realization of the beam a good approximation, and admits the possibility of observing light-atom angular momentum transfer.

In the reported clouds, the range of initial conditions of the atoms is: $0.01 \lambda \leq \rho \leq 2.6 \lambda ; 0.0001 \lambda \leq z \leq 0.001 \lambda$; $-0.0001 \lambda \Gamma \leq \dot{\rho} \leq 0.0001 \lambda \Gamma ; 0.0001 \Gamma \leq \dot{\varphi} \leq 0.00015 \Gamma ;-0.0025 \lambda \Gamma \leq \dot{z} \leq-0.001 \lambda \Gamma$ with the the initial kinetic energies ranging from $\sim 5 \mu K$ to $\sim 30 \mu K$.

Case 1. Single rotating Bessel beam.

The optical potential energy linked to the conservative factor $\vec{\alpha}$, in this case consists of annular potential wells, corresponding to the concentric bright rings of the intensity distribution of the BB. An atom trapped in one of the bright rings of the beam oscillates in the radial direction around the minimum of the potential energy with an amplitude that depends on its initial position and velocity. Along the $z$ axis, the atom is subjected to the gravity force and to the dissipative term $\vec{\beta}$ of the optical force, associated with the phase of the light field $\vec{\beta}=\vec{\nabla} \phi$. In a FORT with the parameters given above, in this case, the dissipative effects have small influence on the atom's motion. Hence, the gravity force dominates the evolution along the z-axis and the transfer of orbital angular momentum from the beam to the atom is also negligible. If the detuning were smaller, the effect of the dissipative forces would be larger, and the optical acceleration along the azimuthal and axial directions would eventually become noticeable.

Figure 5 illustrates the typical trajectories for ten atoms taken from the atomic cloud described above and downloaded in a second order $\mathrm{BB}$ at $z \simeq 0$. Although the light beam is propagating upwards, the atoms move downwards due to their initial negative velocities and to the acceleration of gravity. Gravity also helps to keep the atoms stably trapped within the beam profile in the transverse direction, even if the initial radial position of an atom is close to the axial node $(\rho \sim 0.01 \lambda)$. In contrast, we have verified that some atoms would escape in the radial direction if the atomic cloud were considered with similar initial conditions but with positive sign of the 

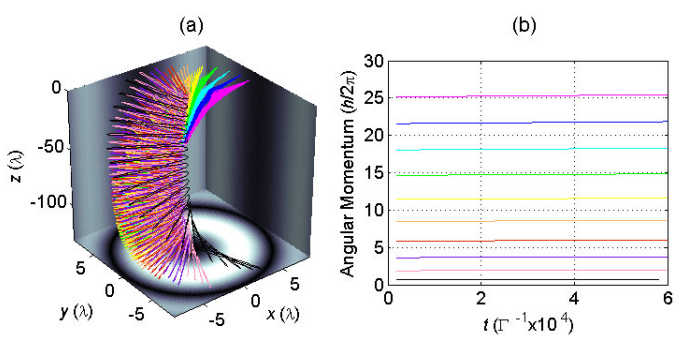

FIG. 5: (a) Spatial paths of ten atoms representing the atomic cloud described in the text moving within a rotating BB. The topological charge of the BB is $m=2$ (positive helicity), and it is propagating along the positive $z$ axis (upwards direction); the axial and transverse components of its wave vector are $k_{z}=0.995 k$ and $k_{\perp}=0.0999 k$. The wavelength of the light is $\lambda=862 \mathrm{~nm}$, which is the length unit in the plots. The smallest value of the kinetic energy and the largest absolute value of the potential energy correspond to the magenta path, whereas the opposite occurs for the black path. The starting point of all the paths is the plane $z \simeq 0$. Notice the scale differences between the three spatial axes. (b) Angular momentum as a function of time for the same atoms. The range of initial conditions of the atoms in the analyzed cloud is: $0.01 \lambda \leq \rho \leq 2.6 \lambda$; $0.0001 \lambda \leq z \leq 0.001 \lambda ;-0.0001 \lambda \Gamma \leq \dot{\rho} \leq 0.0001 \lambda \Gamma ; 0.0001 \Gamma \leq \dot{\varphi} \leq 0.00015 \Gamma,-0.0025 \lambda \Gamma \leq \dot{z} \leq-0.001 \lambda \Gamma)$ with the the initial kinetic energies ranging from $\sim 5 \mu K$ to $\sim 30 \mu K$. The colors of the paths in (a) are directly correlated to the angular momentum in (b).

z-component of the velocity. In Fig. 5(b), we also show the angular momentum as a function of time for each of the atoms illustrated in Fig. 5(a). In all the cases, the angular momentum remains practically equal to its initial value.

Single rotating BBs have been proposed before as guides for cold atoms [6, 7] though no semiclassical calculations were reported. Here, we have considered a red detuned system and found that a BB may indeed be used as an atom guide, in this case.

Case 2. Twisted helical lattice.

The intensity distribution for this case is illustrated in Fig. 22. A transverse cross section looks like a stationary $\mathrm{BB}$, but it is rotating as a whole along the $z$ axis, completing a revolution in a distance of $|m| \lambda_{z}$. For a red detuned lattice, the potential energy minima correspond to a set of $2|m|$ twisted intertwined pipes between each pair of radial nodes, along which the atoms can be guided in independent channels. This light configuration is analogous to that proposed by Bhattacharya [18 for LG beams. However, for BB beams, the propagation invariance introduces additional features for atom guiding with respect to LG beams.

Since the axial and azimuthal variables appear in the combination $\left(m \varphi+k_{z} z\right)$, the light field amplitude has a well defined helicity. In absence of gravity, it is expected that an atom initially moving with the same helicity than that of the light pattern will preserve it, although its angular momentum may change in magnitude. Otherwise, i. e., if an atom has an initial motion with different helicity than that of the light pattern, the optical force might be able to change the atoms helicity. We have verified this fact numerically taking into account gravity effects. This is illustrated in Fig. 6 where: (a) corresponds to loading an atom cloud at the $z \simeq 0$ plane with the parameters mentioned above, so that the atoms initially move downwards and the atoms and the light pattern have the same helicity; (c) corresponds to loading an atom cloud also downwards with the same parameters mentioned above with the exception of $\dot{\varphi}$ whose sign has been reversed, $-0.0015 \Gamma \leq \dot{\varphi} \leq-0.001 \Gamma$, so that initially, atoms and light pattern have opposite helicity. In the latter case, the light force attempts to change the helicity of the atoms by sending them upwards acting against gravity; when it is not able to do so, the atom exhibits a complicated trajectory that may end with its escape. Notice that in both cases (a) and (c), some atoms are able to escape from the radial confinement in the Bessel ring, in particular when they are initially located close to or at a nodal surface; however, they may be eventually trapped at higher radii. The time dependent angular momentum of each atom in both clouds has strong oscillations with an increasing average value, as illustrated in Fig. 6(b) and (d). For atoms with a helicity coinciding with that of the light pattern, an angular momentum average that starts being $10 \hbar$, as in Case 1 , ends with values up to $5 \times 10^{2} \hbar$ for $t \sim 10^{5} \Gamma^{-1}$.

These results show that: (i) twisted helical beams act as wave guides with intertwined channels that determine the rotation direction of radially trapped atoms, and (ii) a significant amount of angular momentum can be transferred 

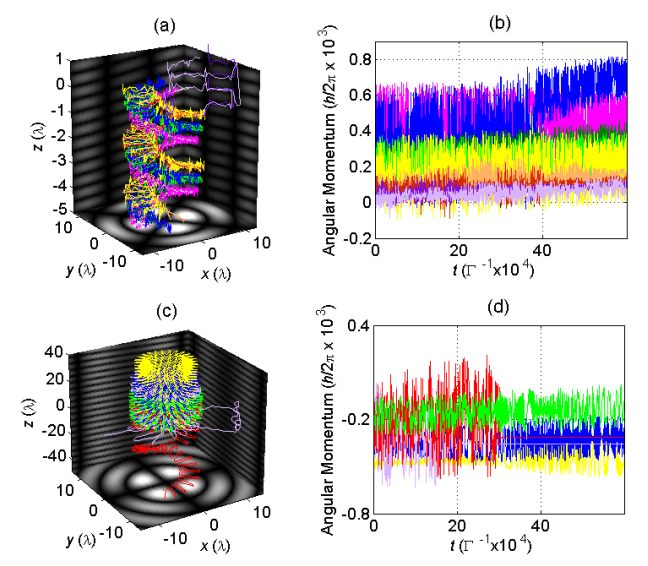

FIG. 6: (a) Illustrative examples of the spatial trajectory and (b) angular momentum of atoms moving within a twisted helical Bessel lattice generated in the way described in Fig. 2. The parameters of the two superimposing beams and the range of initial conditions of the atoms in the analyzed cloud are the same as those of Fig. 5 so that the atoms motion is initially downwards and has the same helicity as the light pattern. In Figure (a) the time interval corresponds to $0<T<0.5 \times 10^{4} \Gamma^{-1}$. Figure (c) shows illustrative examples of the spatial trajectory and (d) angular momentum of atoms moving within the same twisted Bessel lattice than in (a); the initial conditions of the atoms in the analyzed cloud are the same as those used in (a) but with the angular velocity $\dot{\varphi}$ reversed. Notice the scale difference in the axial coordinate in figures (a) and (c). The colors of the paths in (a) and (c) are directly correlated to the angular momentum in (b) and (d).
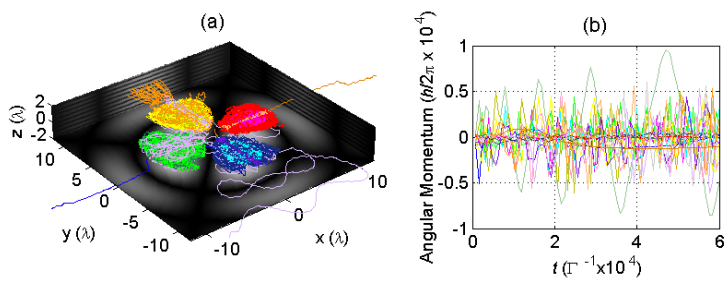

FIG. 7: (a) Spatial path of atoms moving within a 3D Bessel lattice generated by the superposition of two stationary BBs of second order propagating in opposite directions, as described in Fig. 3. (b) Angular momentum as a function of time for the same atoms. The parameters of the two superimposing beams and the range of initial conditions of the atoms in the analyzed cloud are the same as those of Fig. 5 The colors of the paths in (a) are directly correlated to the angular momentum in (b).

to atoms using this beam configuration.

Case 3. 3D stationary circular lattice.

The intensity distribution of this lattice (Fig. 3) corresponds to a set of individual 'potential cages' (potential wells in all the three spatial dimensions) distributed around in a coordinate system with circular cylindrical geometry. Nodal surfaces define the limits of the potential cages. The numerical simulation with the cloud described above shows that: (i) atoms initially located at a nodal surface have a high probability of escaping from the lattice due to a lack of potential energy, particularly when they are very close to the axis of symmetry of the beam; (ii) for atoms initially within a cage, so that their total initial energy is negative, the trapping in this lattice results very robust, regardless of the direction of its initial momentum and in spite of the presence of gravity. In some cases, depending on its initial position and velocity, an atom may tunnel from one cage to the next one either along the axial or the azimuthal direction, while it keeps confined in the other directions. In the latter case, the atom may stay trapped in a transverse plane, going around the whole beam circumference. Illustrative examples of trajectories for the atomic cloud described above are given in Fig. 7.

In Fig. 7, we also observe large fluctuations of the orbital angular momentum $L_{z}$. By comparing with the results 

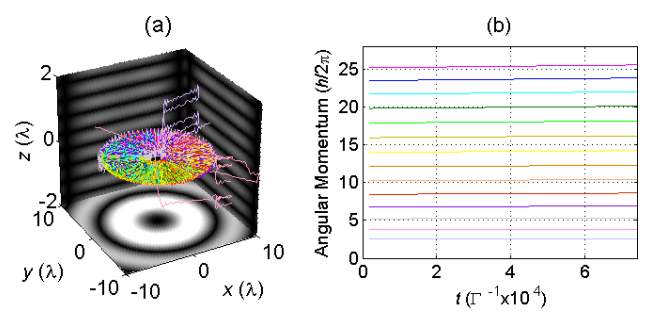

FIG. 8: (a) Spatial path of atoms moving within a toroidal Bessel lattice generated by the superposition of rotating BBs of second order propagating in opposite directions, as described in Fig. 4(b) Angular momentum as a function of time for the same atoms. The parameters of the two superimposing beams and the range of initial conditions of the atoms in the analyzed cloud are the same as those of Fig. 5 The colors of the paths in (a) are directly correlated to the angular momentum in (b).

for the twisted helical configuration, Fig. 6, we notice that the stationary condition in angular and axial directions increases the efficiency of angular momentum transfer in at least one order of magnitude. Notice that in this case, the mean axis of rotation of each atomic trajectory is located in the cages, so that it does not coincide with the axis of the beam.

Based on the high angular momentum oscillations, we consider that this kind of lattice might be especially useful in the generation of vortices in degenerate gases. Notice as well that it would be a more appropriate choice for the studies of quasi one dimensional systems with periodic boundary conditions along the azimuthal direction proposed by Amico and coworkers 21, since the scheme they proposed of interfering a plane wave with a Laguerre-Gaussian beam would give rise to spiral fringes 33 rather than localized spots as in this case. Furthermore, in a 3D stationary Bessel lattice the axial confinement is achieved all-optically instead of magneto-optically. In addition, this lattice could be also a suitable choice for studies of atomic wave function interference between components that rotate in opposite directions.

Case 4. Toroidal train lattice.

The optical potential energy in this case corresponds to a set of toroidal cages aligned along the $z$ axis; the intensity pattern is shown in Fig. 4 . In general, an atom initially located at an antinodal $z$ plane $\left(z=n \lambda_{z} / 2\right)$ will remain trapped in a single torus, waving along the radial and axial directions, and rotating around the beam axis, provided its total initial energy is negative. On the other hand, atoms initially located at a nodal surface may hop to neighbor toroidal traps either one way or the other, while keeping trapped in the radial direction and rotating around the beam axis. This hopping behavior may be exhibited during relatively long time intervals $\left(t \approx 10^{5} \Gamma^{-1}\right)$ before the atom finally escapes. Typical examples of paths followed by atoms of a cloud trapped in this lattice can be seen in Fig. 8(a). If an atom has a non null azimuthal component of its initial velocity, it will remain rotating around the beam axis at practically constant average angular velocity. This can be appreciated from Fig. 8(b), where the angular momenta of the different atoms remain almost constant and have the same order of magnitude than in the case 1, of the propagating rotating $\mathrm{BB}$, which is much smaller than in the other two cases studied here.

\section{3D ATOM CIRCUITS WITH COMBINATION OF BESSEL LATTICES}

With a clear picture of the mechanical behavior of atoms in the different light fields we have discussed so far, we are in a position to elucidate a more sophisticated application. By alternating the operation of different lattices in an appropriated combination, it is possible to create what we will call 'atom loops'. These loops can either be open or 'closed '. By a 'closed' loop we do not mean, of course, that the atom will come back to its initial position, but rather to approximately the same spatial region.

For instance, consider a cloud of atoms downloaded in a toroidal train lattice. After a transitory time, we obtain a steady cloud of atoms trapped in the radial and axial direction moving with essentially their initial angular momentum. By applying the twisted helical BB, they will move downwards or upwards depending on the helicity of the beam and the direction of the atomic azimuthal velocity. In general, the twisted helical BB preserves the radial trapping and modifies the atomic angular momentum $L_{z}$. Now, by turning on a toroidal train lattice just after the twisted helical BB is turned off, the atoms will again be axially trapped. If most of the atoms in the first toroidal lattice moved nearby the $z=0$ plane, in the final configuration, we expect that most atoms rotating in toroidal cages with 


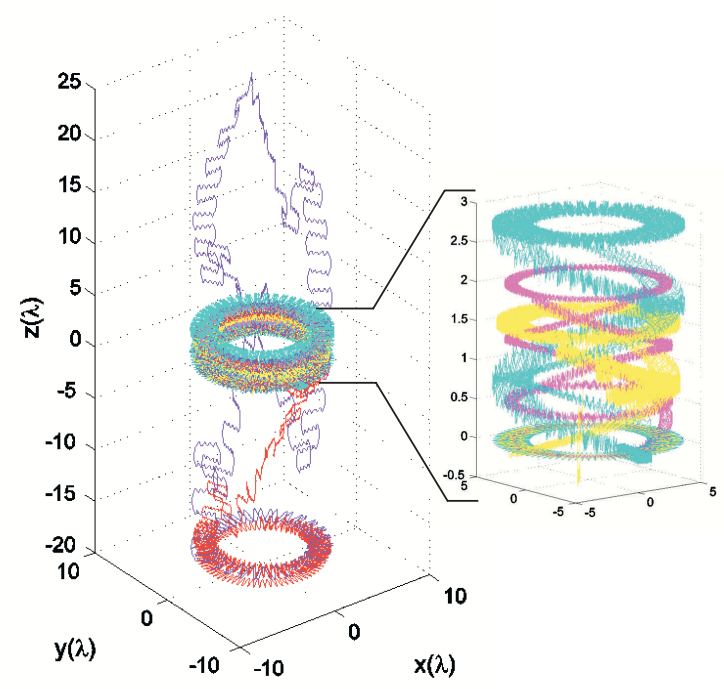

FIG. 9: Some illustrative closed and open atom loops obtained by the effect of five different light fields operated consecutively: (1) toroidal; (2) twisted helical; (3) toroidal; (4) twisted helical with opposite helicity than in (2); (5) toroidal. The general parameters of the light fields correspond to those in Fig. [6] and (8). The initial average kinetic energy is $<K_{i n}>=10 \mu \mathrm{K}$ and the initial angular momentum is $\left\langle\left|L_{z}\right|>50 \hbar\right.$. The application times of each step were optimized to obtain a closed loop for atoms with initial conditions close to those of the blue trajectory. Most of the atoms performed trajectories like the red or purple ones, that is, open loops since their final trajectory is not close to the initial one. However, radial looses were not too frequent during the whole procedure (about 15\%) and most of them occur in the first three steps.

$z>0$ will have an opposite angular momentum $L_{z}$ to those rotating in cages with $z<0$. We confirmed these ideas by performing several numerical simulations of the process. For instance, consider an atomic cloud with an initial average kinetic energy $\left\langle K_{\text {in }}>\approx 10 \mu \mathrm{K}\right.$ and an initial angular momentum average $\left\langle\left|L_{z}\right|>\approx 50 \hbar\right.$. The twisted helical $\mathrm{BB}$ is applied during a time interval $\Delta T=5 \times 10^{4} \Gamma^{-1}$. In the final configuration, in each toroidal cage, $\sim 85 \%$ of the trapped atoms had a common direction of rotation about the $z$ axis. This direction was opposite for $z>0$ and for $z<0$. During the process, $10 \%$ of the atoms were radially lost.

With the current technology of spatial light modulators (SLM), the switching among different options of optical lattices may be performed at reasonably high speeds, limited only by the response time of the specific light modulation device. An experimental study on the interactive generation and switching of the light patterns studied here will be reported elsewhere. Here, we assume valid a sudden approximation in which the atoms do not modify their state of motion during the switching.

We analyzed other loops. As expected, in all cases, the higher the number of steps to obtain a predesigned path, the lower the number of atoms in a cloud that are able to follow it. This can become an advantage of the procedure when the purpose is to select atoms with predetermined mechanical parameters.

Let us consider the following five step circuit: step (1) an atom cloud is trapped in a toroidal cage for a given time interval; step (2) a twisted helical BB is applied with the proper helicity to send the atoms upwards (downwards) if $\dot{\varphi}>0(\dot{\varphi}<0)$; step (3) their are trapped again in a toroidal cage; step (4) they are sent downwards (upwards) using a twisted helical BB with opposite $k_{z}$ than in step (2); step (5) their are trapped again by a toroidal cage. This circuit admits the possibility of obtaining closed atomic loops. This is illustrated in Fig. 9 for an atomic cloud that had an initial average kinetic energy $\left\langle K_{\text {in }}>\approx 10 \mu \mathrm{K}\right.$ and an initial angular momentum $\left\langle\left|L_{z}\right|>\approx 50 \hbar\right.$. The application time intervals for each step were optimized to obtain a closed loop for a small, $5 \%$, subset of atoms corresponding to those with the larger initial radial position $(R \sim 6 \lambda)$. In the procedure, we observed that most radial loses occur at the first three steps. After that about $10 \%$ of the trajectories corresponded to closed loops. All those atoms had the same direction of rotation.

Figure 9 illustrates just an example of a loop, but other combinations can be explored for different purposes. Notice that atoms with preselected energies and momenta could be guided in different directions, so that circuits could be designed with the possibility of performing atom interference experiments. 


\section{CONCLUSIONS}

We have analyzed the dynamical behavior of an atomic cloud moving under the action of four different configurations of light fields with circular cylindrical geometry: a propagating-rotating Bessel beam of order $m$, a twisted helical lattice or twisted helical field, a 3D stationary circular lattice, and a toroidal train lattice. We presented the fundamental equation for the optical force, based on references [25] and [32], and gave the specific expressions for the conservative $\vec{\alpha}$ and dissipative $\vec{\beta}$ terms of the force in each of the cases under study. In section 4 , we discussed the numerical results, case by case, of the solution of the motion equations for the atom, for a red detuned far-off-resonance system. We found that the single rotating Bessel beam and the twisted helical lattice can be used to guide atoms, in the latter case along $2|m|$ separate channels, whereas the 3D stationary circular lattice and the toroidal train lattice can be applied to obtain 3D confinement within a small region of the space. The twisted helical lattice can be used to select atomic helicities and gives rise to strong angular momentum oscillations. The 3D stationary circular lattice define a mean rotation axis for the atomic trajectories located at each potential cage. Finally, on the basis of our numerical results, we proposed an application consisting of the consecutive operation of the different options of light fields studied here, in order to create atom loops in predesigned ways by all-optical means.

Even when we have restricted our analysis here to the case of Bessel modes, it is worth to appreciate that, in the paraxial versions, all the lattices or light fields discussed above would have an analogous in terms of Laguerre-Gaussian laser modes, which might be easier to generate experimentally. In that case, however, beam spreading on propagation should be taken into account; the waist plane of the interfering beams should coincide and the alignment may become an issue. In general, regardless of the specific form of the radial profile, any beam with circular cylindrical symmetry could be useful for generating similar lattices to those studied here, and the behavior of cold atoms in such lattices is expected to be also analogous to that discussed in this paper. It is worth to mention as well, that in the specific case of Bessel lattices with light irradiance of about $6 \mathrm{~mW} / \mu \mathrm{m}^{2}$, we found that atoms with initial kinetic energies up to $30 \mu \mathrm{K}$ can be trapped not only in the first ring of the Bessel profile, but also in the second and even in the third outer rings.

There are also very interesting features occurring in the cases of near-resonance conditions and blue-detuned systems, that are by themselves worthy of other thorough studies. For instance, Gommers and coworkers considered near-resonance conventional lattices to experimentally generate an atomic ratchet [34]; the lattices studied here may represent very attractive novel options for this purpose, since one can generate quasi periodic systems with open boundary conditions along the $z$ axis, but also with closed open boundary conditions along the azimuthal coordinate, as well as explore ratchet systems with new geometries. Thus, a possibility for performing novel studies on stochastic dynamics in atomic systems is opened both classically and quantum mechanically since atomic temperatures will define the proper dynamics. A quantum description of the dynamics of atoms in cylindrical beams will be reported elsewhere.

Acknowledgements. R. J. thanks useful discussions to Prof. J. Recámier. This work was partially supported by DGAPA-UNAM, projects IN103103 and IN115307.

[1] J. D. Miller, R. A. Cline, and D. J. Heinzen, Phys. Rev. A 47, R4567 (1993).

[2] P. S. Jessen, I. H. Deutsch, Adv. Atm. Mol. Opt. Phys. 37, 95 (1996).

[3] E. Cornell, J. of Research of the NIST 101, 419 (1996).

[4] S. Inouye, T. Pfau, S. Gupta, A. P. Chikkatur, A. Gorlitz, D. E. Pritchard, W. Ketterle, Nature 402, 641 (1999).

[5] J. Durnin, J. J. Miceli, and J. H. Eberly, Phys. Rev. Lett. 58, 1499 (1987).

[6] J. Arlt, T. Hitomi, and K. Dholakia, Appl. Phys. B-Lasers Opt. 71, 549 (2000).

[7] J. Arlt, K. Dholakia, J. Soneson, and E. M. Wright, Phys. Rev. A 63, 063602 (2001).

[8] B. M. Rodríguez-Lara and R. Jáuregui, Phys. Rev. A 78, 033813 (2008).

[9] L. Allen, M. W. Beijersbergen, R. J. C. Spreeuw, and J. P. Woerdman, Phys. Rev. A 45, 8185 (1992).

[10] M. Babiker, C. R. Bennett, D. L. Andrews, and L.C. Davila-Romero, Phys. Rev. Lett. 89, 143601 (2002).

[11] K. Volke-Sepúlveda, V. Garcés-Chávez, S. Chávez-Cerda, J. Arlt and K. Dholakia, J. Opt. B: Quantum Semiclass. Opt. 4: S82 (2002).

[12] A. Alexandrescu , D. Cojoc, E. Di Fabrizio, Phys. Rev. Lett. 96, 243001 (2006).

[13] R. Jáuregui, Phys. Rev. A 70 ,033415 (2004).

[14] R. Jáuregui and S. Hacyan, Phys. Rev. A 71, 033411 (2005).

[15] J. W. R. Tabosa and D. V. Petrov, Phys. Rev. Lett. 83, 4967 (1999).

[16] M. F. Andersen, C. Ryu, P. Clade, V. Natarajan, A. Vaziri, K. Helmerson, W. D. Phillips, Phys. Rev. Lett. 97, 170406 (2006). 
[17] H. L. Haroutyunyan, G. Nienhuis, Phys. Rev. A 70, 063408 (2004).

[18] M. Bhattacharya, Opt. Commun. 279, 219 (2007).

[19] P. Exner, M. Fraas, arXiv: 0704.2770vl [quant-ph], 2007.

[20] G. S. Paraoanu, Phys. Rev. A 67, 023607 (2003).

[21] L. Amico, A. Osterloh, and F. Cataliotti, Phys. Rev. Lett. 95, 063201 (2005).

[22] S. E. Olson, M. L. Terraciano, M. Bashkansky, and F. K. Fatemi, Phys. Rev. A 76, 061404R (2007).

[23] S. Schwartz, M. Cozzini, C. Menotti, I. Carusotto, P. Bouyer and S. Stringari, New J. of Phys. 8, 162 (2006).

[24] B. M. Peden, R. Bhat, M. Kramer and M. J. Holland, J. Phys. B: At. Mol. Opt. Phys. 40, 3725 (2007).

[25] J. P. Gordon and A. Ashkin, Phys. Rev. A. 21, 1606 (1980).

[26] S. M. Barnett and L. Allen, Opt. Commun. 110, 670 (1994).

[27] K. Volke-Sepúlveda and E. Ley-Koo, J. Opt. A: Pure Appl. Opt. 8, 867 (2006).

[28] A superposition of TE and TM modes with equal weights $\mathbf{E}_{m}^{( \pm)}=E_{0}^{\prime}\left(\mathbf{E}_{m+1}^{(T M)} \pm i \mathbf{E}_{m+1}^{(T E)}\right)$, corresponds to photons with a helicity, $i$. e., a projection of the spin angular momentum along the $z$ axis of $\pm \hbar k_{z} c / \omega$. In the paraxial limit, this superposition coincides with the definition of circular polarized beams through Eqs. (5).

[29] J. Arlt and K. Dholakia, Opt. Commun. 177, 297 (2000).

[30] A. Vasara, J. Turunen and A. Friberg, J. Opt. Soc. Am. A 6, 1748 (1989).

[31] J. A. Davis, J. Guertin, and D. M. Cottrell, Appl. Opt. 32, 6368 (1993).

[32] V.I. Balykin, V. G. Minogin, V. S. Letokhov, Rep. Prog. Phys. 63, 1429 (2000).

[33] M.P. MacDonald, K. Volke-Sepúlveda, L. Paterson, J. Arlt, W. Sibbett and K. Dholakia, Opt. Commun. 201, 21 (2002).

[34] R. Gommers, S. Denisov, and F. Renzoni, Phys. Rev. Lett. 96, 240604 (2006). 